\title{
ON THE EFFECT OF IMAGE BRIGHTNESS AND CONTRAST NONUNIFORMITY ON STATISTICAL TEXTURE PARAMETERS
}

\author{
Andrzej MATERKA*, Michał STRZELECKI*
}

\begin{abstract}
Computerized texture analysis characterizes spatial patterns of image intensity, which originate in the structure of tissues. However, a number of texture descriptors also depend on local average image intensity and/or contrast. This variations, known as image nonuniformity (inhomogeneity) artefacts often occur, e.g. in MRI. Their presence may lead to errors in tissue description. This unwanted effect is explained in this paper using statistical texture descriptors applied for MRI slices of a normal and fibrotic liver. To reduce the errors, correction of image spatial nonuniformity prior to texture analysis is performed. The issue of sensitivity of popular texture parameters to image nonuniformities is discussed. It is illustrated by classification examples of natural Brodatz textures, digitally modified to account for inhomogeneities - modeled as smooth variations of image intensity and contrast. A set of texture features is identified which represent certain immunity to image inhomogeneities.
\end{abstract}

Keywords: texture analysis, magnetic resonance imaging, image normalization, gray level nonuniformity correction, texture features sensitivity

\section{Introduction}

\subsection{Problem definition}

Interest in texture analysis and its applications to magnetic resonance images (MRI) is constantly growing in the recent years [23]. The texture descriptors provide a rich source of information, computed to characterize spatial patterns of the image intensity that originate in the structure of tissues. Computerized texture analysis is becoming a significant aid to image quantification - for more accurate, reliable and objective medical diagnosis. Although the

\footnotetext{
* Institute of Electronics, Lodz University of Technology, Wolczanska 211/215, 90-924
} Lodz, Poland 
notion of "texture" does not have any general intuitive definition, humans easily perceive image regions of different surface-like appearance. The recognized texture attributes such as "smoothness", "roughness", "granularity", "directionality", etc. [4] have their origin in the physical microstructure of tissues and organs that are visualized in the image [2], [1].

As an extension of the traditional quantitative image analysis based on voxel intensity, texture analysis (TA) deals with intensity patterns. For example, it is known that there are two main symptoms of osteoporosis - reduced bone mass density (BMD) and changes in bone microarchitecture. The latter can be quantified by calculation of texture parameters local image statistics that reflect the presence of image patterns made by the mesh of bone trabeculae. Combination of the BMD and appropriate texture parameter values is a better predictor of the fracture risk than the BMD value alone [23]. The average thickness, number or orientation (anisotropy) of bone trabeculae can be evaluated based on image region. Their values can indicate osteoporotic changes in the bone.

Numerical analysis of image texture has been a topic of active research since the sixties of the past century [26]. A whole-body MR scanner can now produce sub-millimeter resolution images that simultaneously contain information about the external shape of large organs and details of the internal tissues, at a sufficient signal-to-noise ratio (SNR). This makes texture analysis feasible and contributes to increasing interest in clinical applications of MRI TA methods [8], [26]. A "renaissance" of this methodology is now observed [6].

The main issue in TA is calculation of numerical descriptors that uniquely characterize intensity patterns, present in 2D or 3D images. The accepted hypothesis for TA is that by examining values of texture descriptors one can draw conclusions about a pathology or disease process. It is assumed that such processes involve changes in tissue structure that result in measurable change of the image texture, e.g. [3].

The texture descriptors (parameters, features) are statistics computed for voxels inside a region of interest (ROI). Thus ROI should be of a large volume, to contain a statistically significant number of elementary texture patterns. Moreover, the image statistics should be stationary - the ROI should be homogeneous in terms of all the relevant image statistics.

Hundreds of different-definition texture parameters can be computed for a given ROI [26]. Since it is not known in advance which parameters will be the best to describe properties of given texture patterns, most of them are usually computed and their subset is then selected based on their best performance for classification [26] or prediction [3].

It was demonstrated that some of the popular texture descriptors depend not only on texture, but also on some other ROI properties, in particular the intensity mean and variance [20]. This is an undesired property, obscuring the actual effect of texture on the descriptor values. As a consequence, ROI normalization in terms of the intensity mean and variance is one of the recommended preprocessing steps, prior to texture descriptor computation. Relevant procedures are available in TA software packages [26].

Still, the ROI normalization does not eliminate yet another residual (but serious) effect, which is the descriptor dependence on locally varying mean intensity. As an example, it is known that MR images are spatially not homogeneous - for many reasons, such as static magnetic field inhomogeneity, imperfections of gradient and RF coils, etc. [23]. Reviews of methods of MRI inhomogeneity correction are available in [23], [2], in the context of image segmentation through intensity thresholding, usually under the assumption of a Gaussian mixture image model. However, to the Authors knowledge, the significance of image uniformity assumption to texture analysis has not been discussed in the literature, although its correction prior to TA has been done in some studies [16]. (Somewhat related, but of different physical origin, the issue of background-independent texture analysis of X-ray mammograms is discussed in [15].) Thus the aim of this paper is to demonstrate that results 
of TA can be very sensitive to image uniformity inside ROI and that without correction, TA may bring "convincing" but, in fact, false results.

Another objective of this work is to perform more detailed discussion of the influence of image inhomegeneities on commonly used texture parameters. This is rather difficult task since these parameters are not defined directly in the image intensity domain. Instead, they are derived from two-dimensional probability distributions (co-occurrence matrix), image transform (energy of wavelet coefficients in subbands), et cetera. Therefore, we adopted some simplified models of the most commonly used texture descriptors to find out the origins of their possible dependency on image local intensity variations.

The discussion carried out in this paper was illustrated by classification examples of 2D visual image textures. This provided an intuitive support originated from wide experience in dealing with various natural textures, which surround us in daily life.

\subsection{Texture features and ROI normalization}

It is pointed out in the seminal paper [9] that "texture" and "tone" are the most important properties of a region of interest in a monochrome image processed by a computer. These properties are not independent concepts. The tone relates to varying shades of gray of image voxels. The texture, in turn, is linked to information about spatial distribution of those tonal variations. Tone and texture are always present in the image; however, one property can dominate over the other. When a small-area ROI has little spatial variation of voxels intensity, the dominant property is the tone. If the variation is large, the dominant property is the texture. The concept of texture analysis suggested in [7] is related to average spatial relationship that the gray tones have to one another. In particular, it is assumed that texture information is adequately specified by a set of gray-tone-spatial-dependence matrices, which are computed for various angular relationships and distances between voxels-in-pair in the image. Many other descriptors are used to characterize the texture [10], [20].

The assumed model of the image for texture analysis is a stationary random field. Its statistical parameters do not change with voxel location. This assumption is usually satisfied by small-area ROIs extracted from MRIs. If, however, the images of the same tissue are measured by different scanners, taken from different parts of an organ, etc., the ROI intensity mean and variance may be, respectively, different between ROIs. Then, one can show that this "first order" (or tone-related variability) may significantly affect many "second-order" texture parameters [20]. Namely, a variety of texture descriptors do indeed describe the texture in quantity, but they also depend on mean ROI tone and variance. To reduce this effect, the normalization is usually done prior to texture parameter computation. One of the frequently used methods, implemented in the popular MaZda TA package [26] makes all ROIs intensity mean equal to 128 and the intensity variance to 30, in an 8-bit gray-level scale.

\subsection{Image model}

Assume now, the region of interest in an image is of relatively large area which contains a homogeneous texture of a slowly varying local first-order statistics of the intensity (tone). Thus the texture itself is considered stationary within ROI - it may represent the structure of an anatomically defined organ or a uniform part of it (either pathological or healthy). 
However, the background intensity or local image contrast change within the region, and the changes can be represented by smooth functions of the voxel coordinates in space.

Such a model represents a typical image measured by a clinical MR scanner in which anatomically/physiologically irrelevant intensity variations exist. These variations may be induced by the nonuniformity of gradient coils, spatially dependent sensitivity of the radiofrequency coils, the image acquisition measurement sequence properties and other factors. The nonuniformities can be corrected prospectively (using phantoms) and/or retrospectively [23], [4]. The image intensity inhomogeneity in MRI is an adverse phenomenon that affects qualitative and quantitative analysis of images. It may significantly affect texture analysis also, as will be demonstrated later.

Consider a class of statistical texture descriptors based on gray-level co-occurrence matrix (GLCM) [9], [10] which are considered effective in many MRI TA tasks. These descriptors are computed as scalars derived from the GLCMs, in turn computed for all pairs of voxels located at a given distance and direction from each other, within ROI.

As the probability entries of the GLCMs are estimated on the basis of the frequency of the gray level transitions, one cannot distinguish whether a particular entry has its origin in texture or in slowly varying tone. Making this distinction is especially difficult in the case of structural tissue changes caused by a disease in an initial stage of its development, where the texture and tone properties are comparable and balanced. One can show other texture descriptors [20] are also sensitive to smooth variations of image intensity (tone). Comprehensive, general analysis of this property is, however, beyond the scope of this paper.

Following the above, it is postulated that in the cases where the model of slowly varying local intensity is applicable, e.g. for relatively large ROI of uniform texture, the intensity variations should be corrected for (either prospectively or retrospectively), to suppress the effect of inhomogeneity artifacts on TA. Magnetic resonance images of healthy and fibrotic liver were chosen to support this recommendation. Results of their texture classification are presented and discussed in the following Sections to illustrate the main points of the issue.

\section{Materials and methods}

\subsection{MR liver images}

Analysed MR liver images represent healthy volunteer and patient with liver fibrosis. They were imaged with breath-hold respiration motion artifact reduction technique, using a 3T MRI device (Philips Achieva) and a T1 weighted pulse sequence (THRIVE iso: ultra-fast gradient echo, TR/TE $=2.76 / 1.36 \mathrm{~ms}, \mathrm{a}=10^{\circ}, \mathrm{FOV}=400 \times 400 \mathrm{~mm}^{2}$, matrix size $=192 \times 192$, isotropic voxel $=2.08 \times 2.08 \times 2.08 \mathrm{~mm}^{3}$ ). For analysis, 13 cross-sections of each case representing middle part of the liver were considered. Sample cross-sections are presented in Fig. 1. The liver disease was diagnosed based on liver biopsy. The images of each type were acquired for a patient and a healthy volunteer with their informed consent, approved by a local ethics committee (courtesy of Dr Jacques de Certaines, within the framework of EU COST B21 action). 

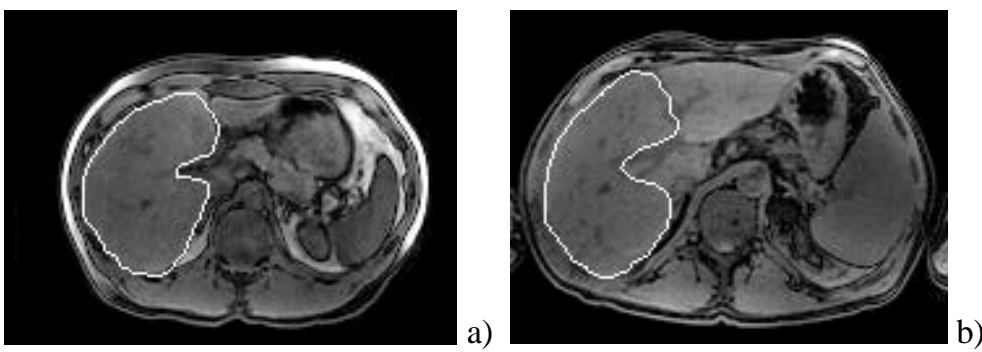

Figure 1. Sample slices of 192x135-pixel MR liver images. White lines delimit ROIs of, respectively, (a) healthy and (b) fibrotic liver

The steps of liver image analysis include ROI definition, feature extraction, feature selection and classification, Fig. 2. Before analysis each ROI was normalized, by calculating mean value $(m)$ and standard deviation $(\sigma)$ of its gray levels. Normalization involved the mean value subtraction and division of the difference by the standard deviation. Subsequently, the ROI gray levels were quantized in the range of $m \pm 3 \sigma$ [26]. Next, for each ROI the texture features were estimated. They were based on the following approaches: cooccurrence matrix, run-length matrix, gradient matrix, autoregressive model, and Haar wavelet transform (WT). A detailed definition of these features is described in [20]. To reduce the number of texture features to most significant ones, the Fisher criterion [22] was applied. Classification based on 1-nearest neighbor classifier (1-NN) was performed using b11 - a part of MaZda software package [26].

A closer look at the liver cross-sections, e.g. those shown in Fig.1, reveals that the average local image intensity is not the same inside different parts of a ROI. In particular, the lower left part of the fibrotic liver image is darker than its upper part. To investigate this effect in quantity, the local average intensity was approximated by a smooth function of image coordinates $x, y$.

In the case of MRI, the average intensity variations are of multiplicative nature, as their source is in spatial variation of the measured RF signal power (e.g. related to $B_{0}$ magnetic field or RF receiver coils nonuniformities). Other modalities, e.g. visual light camera [7] or computed tomography images [1] may feature additive variations as well. To keep the discussion more general, we investigated and compared both cases - additive and multiplicative nonuniformity artifacts and their effects on texture analysis in the presented example. 


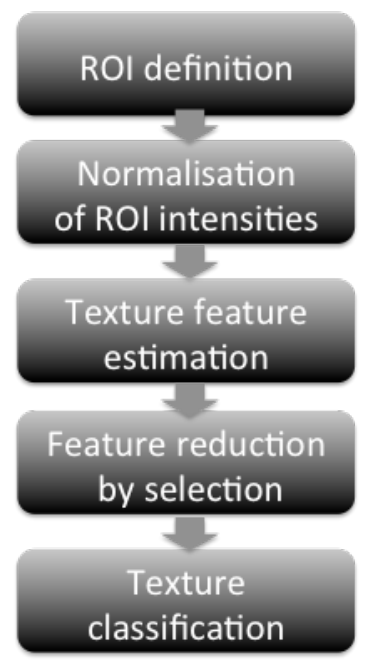

\section{Figure 2. Block diagram of image processing steps}

Before correcting the nonuniformity of an image, its local average intensity $f_{n}\left(x_{n}, y_{n}\right)$ was estimated within square windows centered at nodes of a regular grid inside ROI, $n=1,2, \ldots, N$. The size of the window was compromised between smoothing the texture details inside it and providing a large number of independent windows covering the ROI area. In the considered example, the windows size was set to $5 \times 5$ pixels and their centers were separated by 5 pixels in both, horizontal $(x)$ and vertical $(y)$ directions. Their total number $N$ was varying from about 40 to 80 , depending on a ROI size.

In the case of additive artifacts, the background image intensity inside ROI was modeled by a $3^{\text {rd }}$ order polynomial [19]

$$
\begin{aligned}
& f_{s}(x, y ; a)= \\
& 1+a_{0}+a_{1} x+a_{2} y+a_{3} x^{2}+a_{4} x y+a_{5} y^{2}+a_{6} x^{3}+a_{7} x^{2} y+a_{8} x y^{2}+a_{9} y^{3}
\end{aligned}
$$

such that the local average image intensity inside ROI is estimated as the product of its intensity mean value $m$ and $f_{s}$, as follows

$$
\forall_{(x, y) \in R O I} f_{b}(x, y) \cong m f_{s}\left(x, y ; a^{(a)}\right)
$$

For the multiplicative model artifacts, the image background intensity inside ROI is estimated as the ratio

$$
\forall_{(x, y) \in R O I} f_{b}(x, y) \cong m / f_{s}\left(x, y ; a^{(m)}\right)
$$

The symbols $\boldsymbol{a}^{(a)}$ in (2) and $\boldsymbol{a}^{(m)}$ in (3) denote the parameter vector $\boldsymbol{a}=\left[a_{0}, a_{1}, \ldots, a_{9}\right]$ of the surface (1) used to represent the additive and multiplicative artifacts, respectively. They were estimated for each ROI through minimization of a sum-of-squared error $\varepsilon$ between the local intensity average $f_{n}\left(x_{n}, y_{n}\right)$ and the modeled average, with the use of $f_{s n}(\boldsymbol{a})=f_{s}\left(x_{n}, y_{n} ; \boldsymbol{a}\right)$, for all center coordinates $\left(x_{n}, y_{n}\right)$ of the averaging windows, $n=1,2, \ldots, N$. For additive artifacts, the error function was computed as 


$$
\varepsilon^{(a)}(a)=\sum_{n=1}^{N}\left[f_{n}-m f_{s n}(a)\right]^{2}
$$

where $\boldsymbol{a}=\left[a_{0}, a_{1}, \ldots, a_{9}\right]$ is the vector of unknown parameters.

In the case of multiplicative artifacts model, the error function was defined as

$$
\varepsilon^{(m)}(a)=\sum_{n=1}^{N}\left[f_{n}-m / f_{s n}(a)\right]^{2}
$$

Minimization of (4) can be converted to solving a system of linear equations, while minimization of (5) is a nonlinear problem. In both cases, however, a universal unconstrained Powell minimization routine available in Scipy Python library [11] was used to find the respective parameters $a_{\text {opt }}$ of the model (1) for each ROI.

Finally, for additive artifacts, the intensity of each pixel inside ROI was corrected using the following formula

$$
f^{(a)}(x, y)=f(x, y)-m\left[f_{s}\left(x, y ; a_{o p t}^{(a)}\right)-1\right]
$$

where $a_{o p t}^{(a)}$ is computed through minimization of (4). For the multiplicative model, the corrected image was obtained through

$$
f^{(m)}(x, y)=f(x, y) f_{s}\left(x, y ; a_{o p t}^{(m)}\right)
$$

where $\boldsymbol{a}_{\text {opt }}^{(m)}$ is the argument of minimized (5). As one can notice, if image background intensity is constant within ROI, all the elements of the parameter vector $\boldsymbol{a}$ in (1) are zero. Then, for each image point $(x, y)$ inside ROI, $f_{s}(x, y ; 0)=1$ and, consequently, the images computed with (6) or (7) are simply not corrected - they are equal to original ones in such cases.

\subsection{Brodatz textures}

To illustrate the discussion presented in Section 3, four images representing Brodatz album [5] textures were considered. These 8-bit encoded images with size 256x256 are presented in Fig. 3. Two types of image nonuniformity were considered. First, corresponding to variable intensity, was modeled by additive function shown in Fig. 4a. Second type reflects variable contrast and is represented by function from Fig. $4 \mathrm{~b}$. Both functions were defined using a linear function [23]

$$
g(x, y)=c_{1} x+c_{2} y+c_{3}
$$

where $c_{1}, c_{2}, c_{3}$ are constants - respective model parameters. The degraded texture images are shown in Fig. 5. 

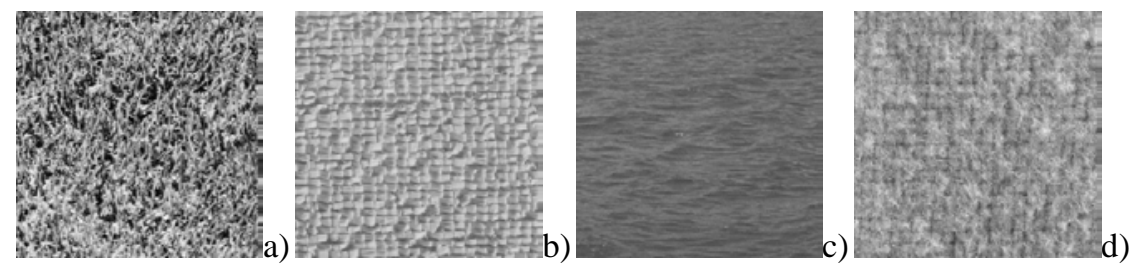

Figure 3. Sample Brodatz textures used for analysis: grass (a), raffia (b), water (c), and wool (d)

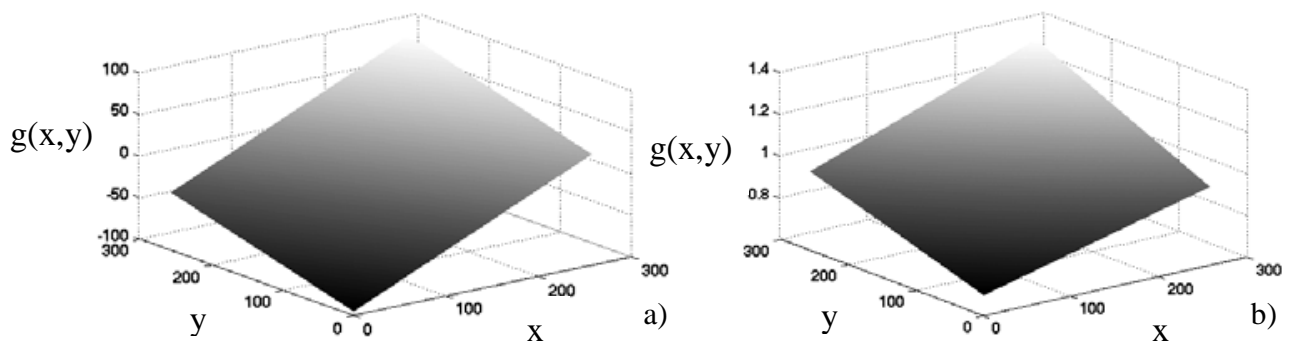

Figure 4. Variable image intensity (a) and contrast (b) models
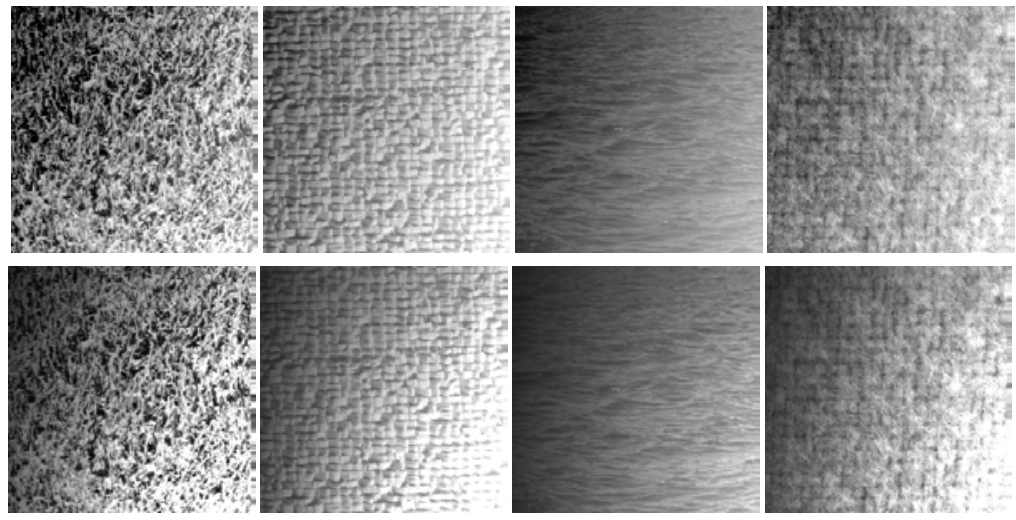

a)

Figure 5. Textures from Fig. 3 degraded by additive (a) and multiplicative (b) nonuniformities

The original and modified Brodatz-album images were analyzed using the same methodology as in the case of liver images, except for ROI definition. Namely, each Brodatz image was split into 16 square-shaped non-overlapping ROIs.

\section{Sensitivity of selected texture parameters to image nonuniformities}

This Section presents a discussion on how commonly used texture features are influenced by variable image intensity mean and contrast. These variations are modeled by additive or 
multiplicative function respectively that modify gray level distribution of the original texture. In most practical situations, the size of the region of interest (ROI), which contains the texture under analysis, is small. This especially happens in cases where the change in texture corresponds to an early stage of a disease affecting the tissue structure. If ROI is small, one can assume the variations of its local average intensity and contrast can be described by a smooth, close-to-linear functions. Examples of such functions (see eqn. (8) in Section 2) are shown in Fig. 4.

Let us consider a linear model of slowly varying local intensity (Fig. 4a) and assume the intensity characterized by this model is quantized using a small number $K$ of quantization intervals. Now, to simplify the discussion, the quantized intensity variation is added to an image of otherwise uniform texture. As an example, Fig. 6a illustrates the image filled with uniform texture whose additive linearly varying local intensity is quantized to four intervals. This simplification does not impose any lack of generality - in the limit the number $K$ can be increased to a value adequate for the local intensity representation.

\subsection{GLCM}

The probability entries of the GLCMs are estimated on the basis of the frequency of the gray level $i$ and $j$ transitions, assuming given interpixel distance and analysis direction [26]. Additive nonuniformity is modeled by a smooth (but stepwise, see above) function that consists of $K$ regions with means $m_{k}$. Adding the stepwise intensity component will cause transformation of an original texture GLCM element $a[i, j]$ into a set of elements $a\left[i+m_{k}, j+m_{k}\right], a\left[i+m_{k}, j+m_{(k+1)}\right], a\left[i+m_{(k+1), j}+m_{k}\right], k=1 \ldots K$. Their values are proportional to respective region area and border length between regions. Sample case is illustrated in Fig. 6 . It presents uniform texture degraded by intensity variation that includes four regions with means $0<m_{1}<m_{2}<m_{3}<m_{4}$ respectively (Fig. 6a). Thus the GLCM entry $a[i, j]$ will be transformed into a number of entries, as shown in Fig. 6b. These elements will be located in the matrix diagonal (gray circles) within $m_{k}$ from $a[i, j]$ (the same concerns off-diagonal GLCM elements). Additionally, new elements will appear that represent pixels located in the region borders: $a\left[i+m_{k}, j+m_{k+1}\right]$ and $a\left[i+m_{k+1}, j+m_{k}\right]$, (white circles in Fig. 6b), distributed on both sides of $a\left[i+m_{k}, j+m_{k}\right]$ The discussed transformation of GLCM can be described as

$$
\begin{gathered}
a[i, j] \rightarrow \bigcup_{\mathrm{k}=1}^{\mathrm{K}} S_{k}\left(a\left[i+m_{k}, j+m_{k}\right]+2 L_{k, k+1} a\left[i+m_{k}, j+m_{k+1}\right]\right) \\
\sum_{k=1}^{K}\left(S_{k}+2 L_{k, k+1}\right)=1
\end{gathered}
$$

where $S_{k}$ and $L_{k, k+1}=L_{k+1, k}$ represent the contribution of corresponding region areas and border lengths in relation to the whole image size respectively, $K$ denotes the number of different regions. For slowly varying intensities one can assume that $m_{k} \approx m_{k+1}$,for every region $k$. This means that translated GLCM elements will create close groups in parallel to matrix diagonal ('tails', as shown in Fig. 6b). Further illustration of these effects will be shown in Section 4.

GLC matrices are constructed for assumed direction $\left(0^{\circ}, 90^{\circ}, 45^{\circ}\right.$ and $\left.135^{\circ}\right)$ and distance between pixels in pairs. For such matrices, a number of texture features can be computed [7]. Let $\left(d_{x}, d_{y}\right)$ be a vector whose elements are respectively the $x$ and $y$ co-ordinate differences between pixels, pairwise. The interpixel distance is defined as $\max \left(d_{1}, d_{2}\right)$, while the direction is generally specified by the vector $\left(d_{x}, d_{y}\right)$. The two numbers $\left(d_{x}, d_{y}\right)$ in brackets 
precede a feature name. For example, “(1,0)Entropy” means that this particular feature was estimated for a GLCM computed on the basis of pixels separated by unit distance in the horizontal direction.
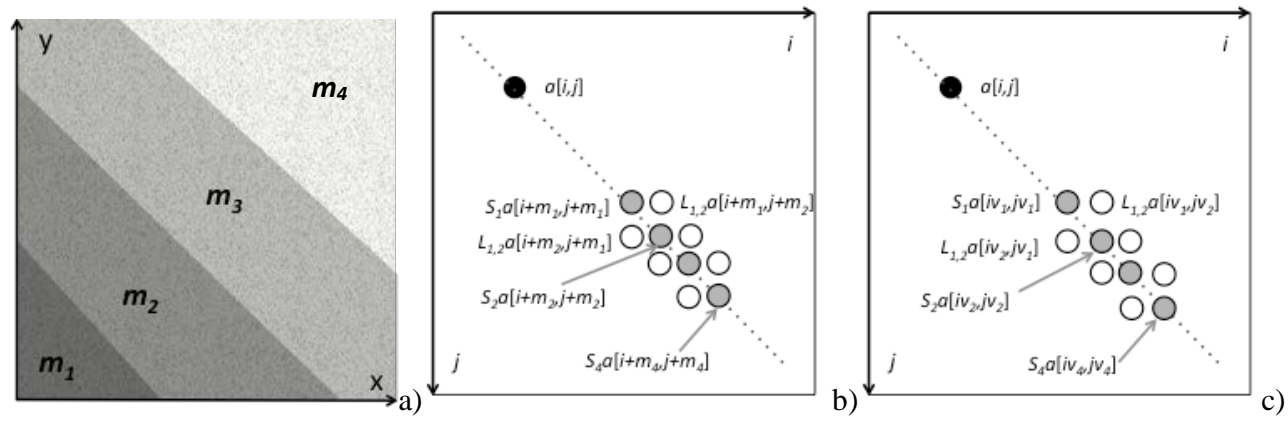

Figure 6. Sample texture with local intensity variation represented by four constant regions with means $m_{1}-m_{4}$ (a). Distribution of GLCM elements: $a[i, j]$ for original texture and after additive (b) and multiplicative (c) distortion.

Some texture parameters defined for the GLCM directly depend on difference between indexes $(i, j)$ of matrix elements. For example, the Contrast feature is defined as follows:

$$
\text { Contrast }=\sum_{n=0}^{G-1} n^{2}\left(\sum_{i=0}^{G-1} \sum_{j=0}^{G-1} a[i, j]\right), \quad n=|i-j|
$$

where $G$ is a number of image gray levels. For $K$-region additive intensity the Contrast descriptor is defined by (11):

$$
\begin{aligned}
\text { Contrast }^{K}=\sum_{k=1}^{K} & n_{k}^{2} \sum_{i=0}^{G-1} \sum_{j=0}^{G-1} S_{k} a[i, j]+2 \sum_{k=1}^{K} n_{k, k+1}^{2} \sum_{i=0}^{G-1} \sum_{j=0}^{G-1} L_{k, k++_{-}} \\
& \approx n^{2} \sum_{i=0}^{G-1} \sum_{j=0}^{G-1} \sum_{k=1}^{K} S_{k} a[i, j] \\
& +n^{2} \sum_{i=0}^{G-1} \sum_{j=0}^{G-1} \sum_{k=1}^{K} 2 L_{k, k+1} a[i, j] \\
& =n^{2} \sum_{i=0}^{G-1} \sum_{j=0}^{G-1} \sum_{k=1}^{K}\left(S_{k}+2 L_{k, k+1}\right) a[i, j]=\text { Contrast }
\end{aligned}
$$

Since $n_{k}=\left|\left(i+m_{k}\right)-\left(j+m_{k}\right)\right|=|i-j|=n$ and, assumed $m_{k} \approx m_{k+1}, n_{k, k+1}=\left|\left(i+m_{k}\right)-\left(j+m_{k+1}\right)\right|=\mid\left(i+m_{k+1}\right)-$ $\left(j+m_{k}\right)|\approx| i-j \mid=n$.

Thus small additive modifications of local intensity will not modify significantly the value of this texture feature. The same conclusion will be valid for some other parameters, such as Sum of Squares, Inverse Difference Moment, Variance and Entropy of Difference Distribution because these parameters also depend on the difference of GLCM element indexes. Thus values of these parameters should not be much sensitive to slow variations of texture intensity.

Multiplicative nonuniformity is also modeled by a smooth (but stepwise) function that consists of $\mathrm{K}$ regions of different intensity multiplication $v_{k}=1+c_{k}$, where $c_{k}$ characterizes the variation of image contrast across regions, $k=1,2, . ., K$. Introducing this variations will transform an original texture GLCM element $a[i, j]$ into a set of elements $a\left[i v_{k}, j v_{k}\right]$, 
$a\left[i v_{k}, j v_{k+1}\right], a\left[i v_{k+1}, j v_{k}\right], k=1 \ldots K$, as in the case of intensity nonuniformity (original image gray levels are modified by multiplying their values by respective local coefficient $v_{k}$ ). Assuming smooth changes of the multiplicative nonuniformity $\left(v_{k} \approx v_{k+1}\right)$, these elements will be also located close to each other. However, due to the index multiplication operation, their clusters will not be located in parallel to the GLCM diagonal. Sample matrix element distribution is shown in Fig. 6c, assuming that original texture was degraded by means of multiplicative nonuniformity modeled by four regions with coefficients $1<v_{1}<v_{2}<v_{3}<v_{4}$ respectively. As a result, the previously discussed GLCM parameters will be more sensitive to local contrast distortion when compared to the additive changes since difference of indexes will not cancel the effect introduced by multiplication $\left(\left|i v_{k}-j v_{k}\right|=v_{k}|i-j| \neq|i-j|\right)$. However, for small contrast changes $\left(v_{k} \approx 1\right)$, these elements will be less sensitive to contrast variation than other ones defined for GLCM, which by definition do not depend on index difference.

Our study revealed also that some GLCM texture parameters which are in general sensitive to multiplicative changes of image intensity, become insensitive to them if the function describing this nonuniformity possesses appropriate symmetry in the image coordinate system.

\subsection{RLM}

RL matrix elements $p[i, r]$ represent the number of times there is a run of length $r$ having grey level $i$, for given direction in the image [10]. Considering similar assumptions as in the case of GLCM discussion, additive image intensity changes will cause two effects. First, RLM entries split into $K$ new ones located in matrix rows indexed by $i+m_{k}$. Also, this new entries shift towards shorter runs $r_{k}$, proportionally to the shape and length of the regions, $r_{1}+\ldots+r_{k}=r$. This is illustrated in Fig. 7 where sample element $p[i, r]$ of original texture split into four ones $p\left[i+m_{k}, r_{k}\right], k=1 \ldots 4$ (assuming variable intensity model with four regions as shown in Fig. 6a). The transition of RLM elements for additive local intensity variation is illustrated by eq. (12):

$$
p[i, r] \rightarrow \bigcup_{k=1}^{K} p\left[i+m_{k}, r_{k}\right], \quad \sum_{k=1}^{K} r_{k}=r
$$

where $r_{k}$ corresponds to new shorter run lengths introduced after split of original element $p[i, r]$.

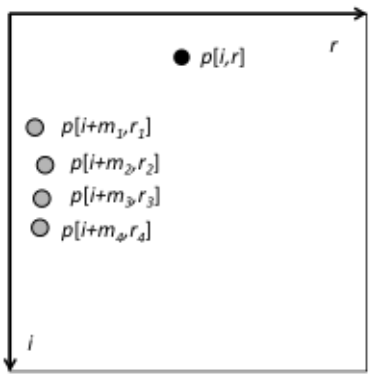

Figure 7. Modification of RLM as a result of variable intensity: original element $p[i, r]$ splits into four ones with shorter runs and intensities altered by $m_{k}$ 
Similar effect will occur for multiplicative degradation, except for the new element locations will depend on the product of $i$ and $m_{k}$. This means that RLM structure will be rather significantly modified, in terms of element distribution and alteration of their values.

\subsection{Wavelet coefficients}

The discrete WT of a signal is based on its successive decomposition into high-pass and lowpass filtered components [17]. Each component is down-sampled by 2 to provide next-scale (lower resolution) signal, for further decomposition with the same filter bank. In the 2D case, the $1 \mathrm{D}$ analysis filter bank is usually first applied to the columns of the image and then applied to the rows of the first-step result. The normalized energy of the image at each scale is typically computed to provide the parameters of the image texture. The MaZda software [24,26] computes Haar-wavelet DWT up to 8 scales with a cascade of high and low-pass filters, resulting in four subbands (low-low, low-high, high-low and high-high) for each scale. This is also reflected in names used for estimated energies of wavelet coefficients. First two letters $(\mathrm{L}$ or $\mathrm{H}$ ) correspond to given subband while a number denotes the scale. For example, “(H,L,2)Wavelet_energy" was estimated for high-low subband at second scale. Due to high-pass filtering, slow variations of image intensity are suppressed, thus one can expect that energies evaluated for these subbands should preserve the information about the texture only. Thus Haar DWT parameters should be resistive against local intensity variation, except for those estimated for low-low subband. The high-pass filtering that partially removes variable illumination is built into image gradient operator, as well. Thus the latter conclusions are practically valid for parameters estimated from gradient matrix in the case of additive variation of texture intensity. Variable contrast will modify image gradient resulting in modification of estimated parameter values.

Considering other texture models (e.g. statistical, like autoregressive), they in general show sensitivity of their parameters to image local intensity and contrast variations.

\section{Results}

\subsection{MR liver images}

First, texture features were computed for all 26 MR liver images used in this experiment. A scatter plot on the best features plane is shown in Fig. 8. As the two clusters are quite compact and well separated, it seems one can distinguish images of a fibrotic liver from images representing the healthy group, with no error.

Bearing in mind, however, that the measured images at hand are apparently not uniform in terms of local average intensity, their corrections were made with the use of additive and multiplicative models. An example of the additively corrected liver images shown in Fig. 1 is presented in Fig. 9. As a matter of fact, the multiplicatively corrected images look the same, at least to the Authors' eyes. 


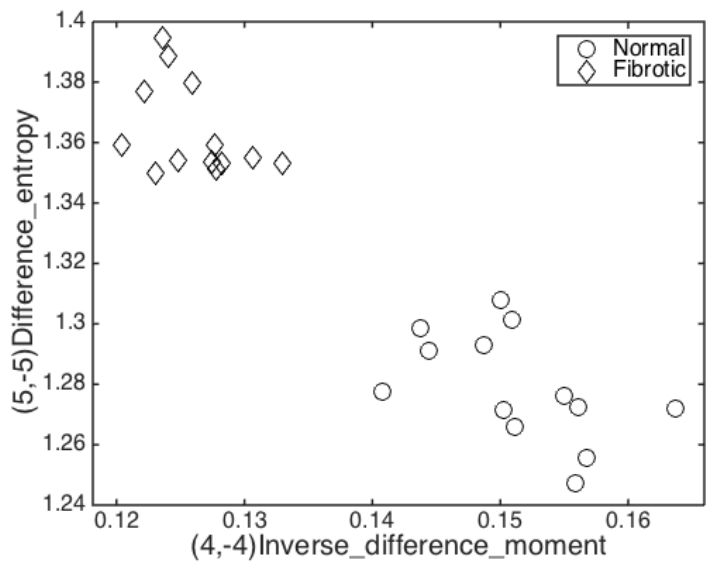

Figure 8. Scatter plot of best discriminating co-occurrence matrix parameters computed for thirteen different slices of normal and fibrotic liver MR image. Fisher coefficient $F=27.1$, misclassification rate $e=0 / 26$.

The texture features were then computed for the corrected images and best of them were selected using the largest Fisher coefficient criterion. The best-feature scatter plots for additively and multiplicatively corrected images are respectively shown in Fig. 10 and Fig. 11. First, one can notice that the best features are different from those selected for the uncorrected images. Moreover, the class clusters for the corrected ones are less compact and relatively closer to each other. Thus the removed average intensity field has had an important contribution to the "nice" class separation shown in Fig. 8.
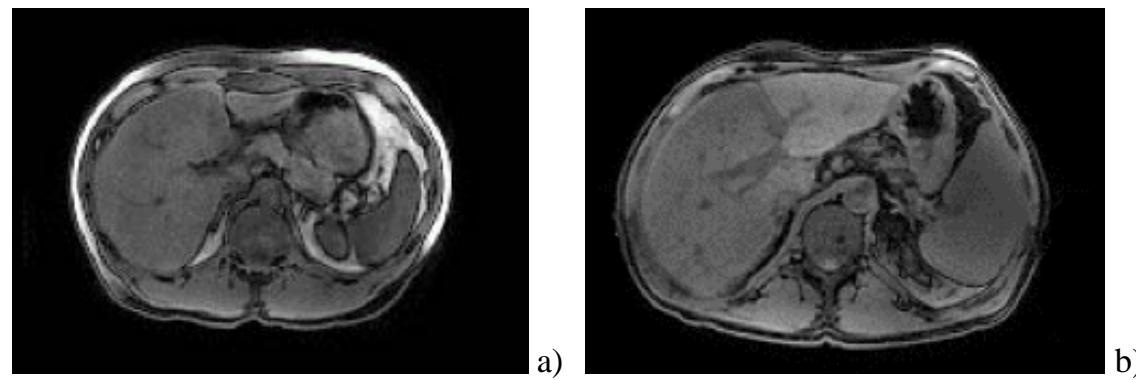

Figure 9. Normal (a) and fibrotic (b) liver images of Fig. 1, corrected using the additive (brightness non-uniformity) model.

Figure 12 shows a distribution of the best features selected for multiplicatively corrected images applied to additively corrected ones and the best features selected for additively corrected liver images applied to multiplicatively corrected ones. Again, good separation of the two texture classes is possible in both cases. Thus the additive and multiplicative corrections produce images of similar "texture content" in the considered case of liver MRI slices. 


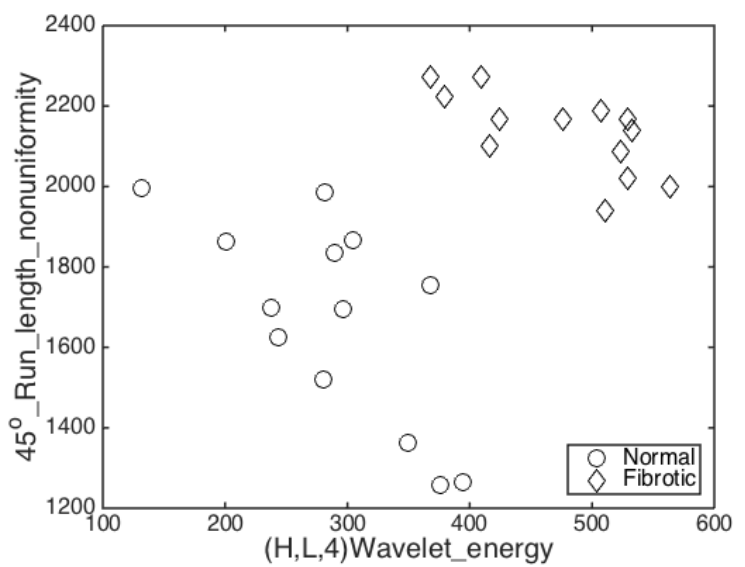

Figure 10. Scatter plot of best discriminating texture parameters computed for additively corrected MR images of normal and fibrotic liver $(F=6.4, e=0 / 26)$.

On the other hand, using the features that best separate the two classes of uncorrected images (see Fig. 9) for discrimination of the corrected images is a failure. This is illustrated in Fig. 13 where the $(4,-4)$ Inverse_difference_moment and $(5,-5)$ Difference_entropy features, computed for additively corrected images, are scatter plotted. Similar effect is observed when these features are used or classification of the multiplicatively corrected images.

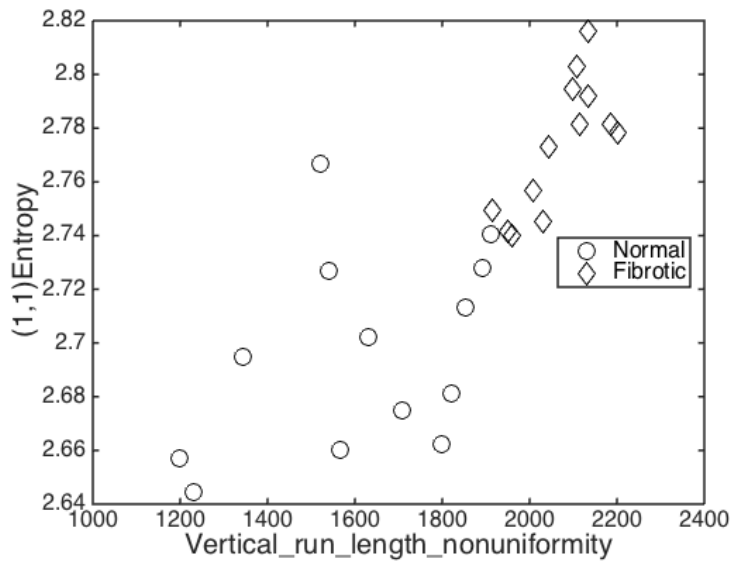

Figure 11. Scatter plot of best texture features for multiplicatively corrected liver images $(F=6.8, e=1 / 26)$. 


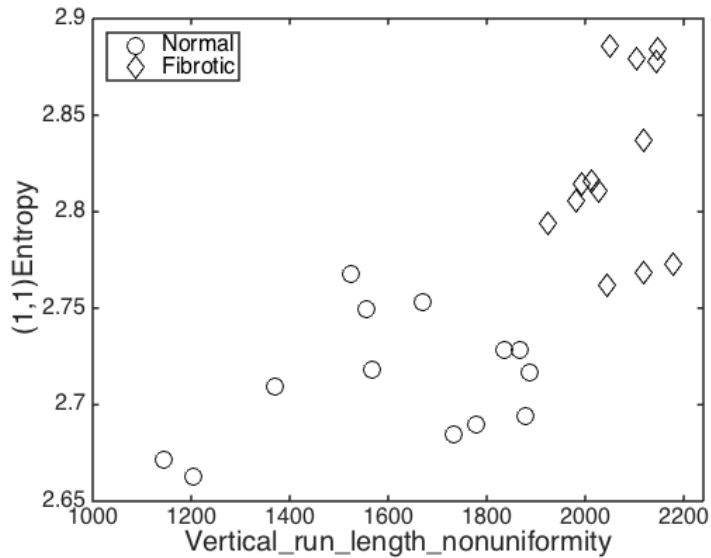

a)

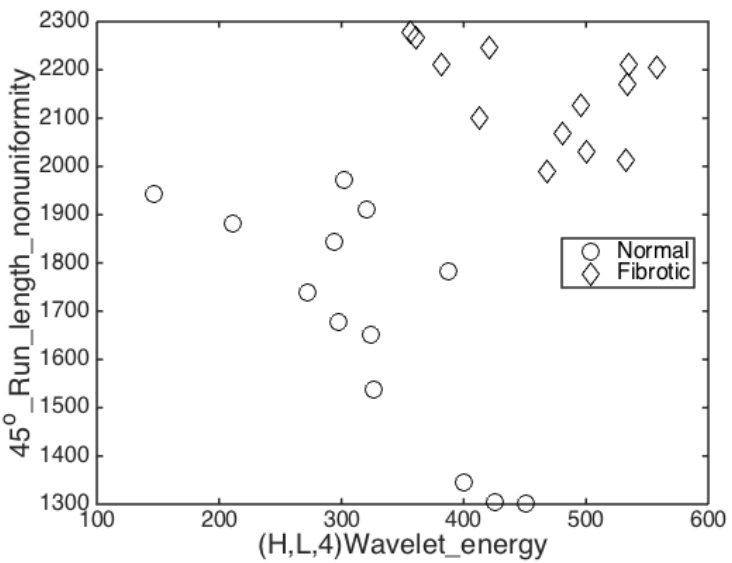

b)

Figure 12. Scatter plot of best texture features for a) multiplicatively corrected liver images applied to additively corrected ones $(F=6.3, e=0 / 26), b)$ additively corrected liver images applied to multiplicatively corrected ones $(F=5.8, e=0 / 26)$. 


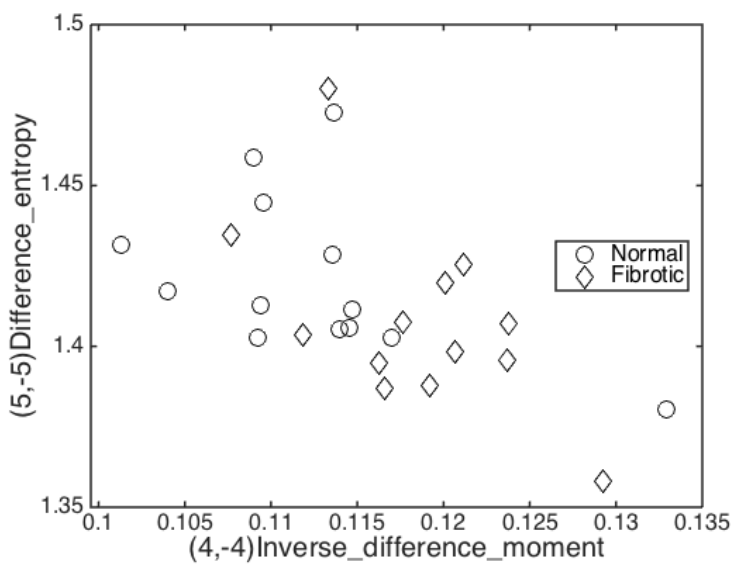

Figure 13. Scatter plot of best texture features for uncorrected liver images applied to additively corrected ones $(F=0.6, e=12 / 26)$.

To evaluate the contribution of the slowly varying local average intensity of the images shown in Fig. 1 to their "texture analysis", the modeled local average intensity images $m f_{s}\left(x, y ; a_{o p t}\right)$ were computed inside ROIs (for the additive correction example), as presented in Fig. 14, respectively for fibrotic and healthy liver. Their texture features were then computed and selected using the largest Fisher coefficient criterion. The scatter plot of the best texture features in this case is shown in Fig. 15a. One can notice, the two classes of liver images can be very well separated, even if there is no image texture information inside ROI. On the other hand, it is rather unlikely that the local average smooth variations of the image intensity are caused by the development of fibrosis. Moreover, they are present in healthy liver as well, but incidentally their surfaces have different shape. Thus the results presented in Fig. 15a wrongly combine two effects - the texture and the image nonuniformity. Similar results were obtained for other example liver images collected within the framework of EU COST B21 Action.
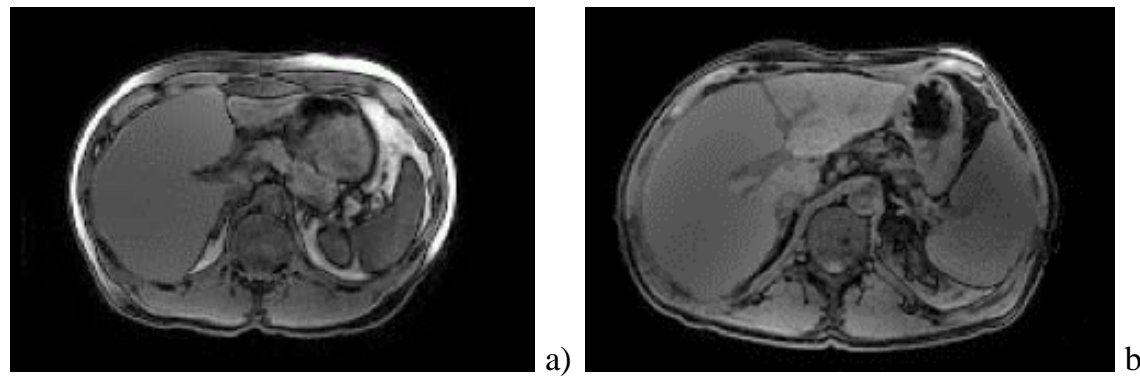

Figure 14. Local average intensity field for additive correction: a) healthy, b) fibrotic liver, respectively corresponding to Fig 1a and Fig. 1 b. 


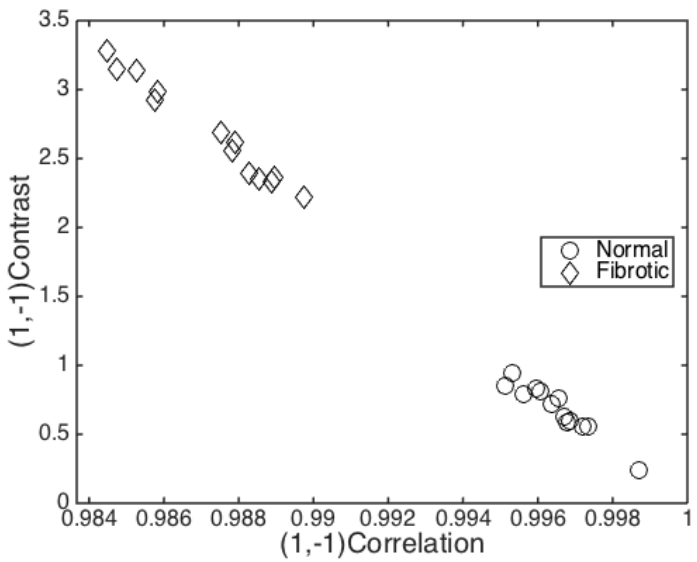

a)

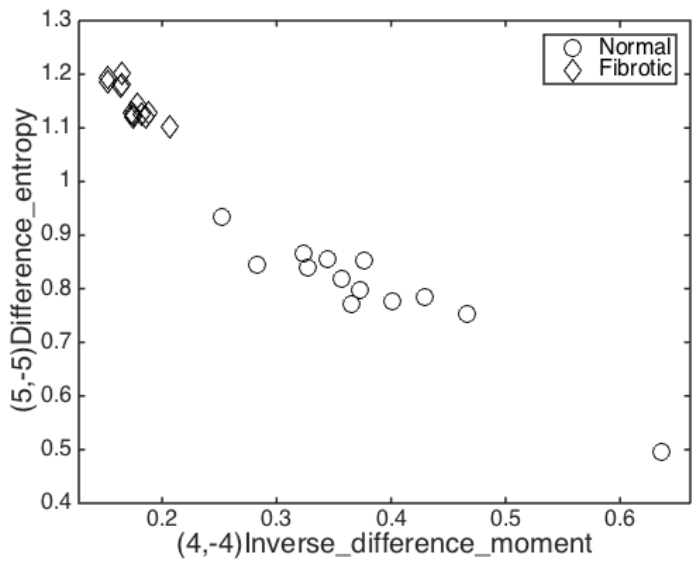

b)

Figure 15. Scatter plot of best texture features a) for the images of local average brightness (for the additive correction fields) $(F=53.0, e=0 / 26), b)$ for uncorrected liver images applied to the images of local average brightness $(F=16.8, e=0 / 26)$.

Finally, the texture features (4,-4)Inverse_difference_moment and (5,-5)Difference_entropy selected as being the best for the uncorrected image classes separation (Fig. 8) were also computed for the additive correction fields, i.e. the images of smoothly varying image intensity. The obtained scatter plots are presented in Fig. 15b. One can see that these features are still very good for the classification of the images with removed texture.

It should be emphasized that the local-average-brightness images contain the smooth correction surfaces only, with the original image content (i.e. texture) removed. To better visualize the properties of the original, corrected and additive-correction images, their examples corresponding to slice \#50 were surface-plotted in Fig. 16. The spatial variations of the image intensity well seen in Fig. 16b are considered as representing the texture patterns. There is no texture in this sense in Fig. 16c, and the texture is superimposed on smooth background variations in Fig. 16a. 

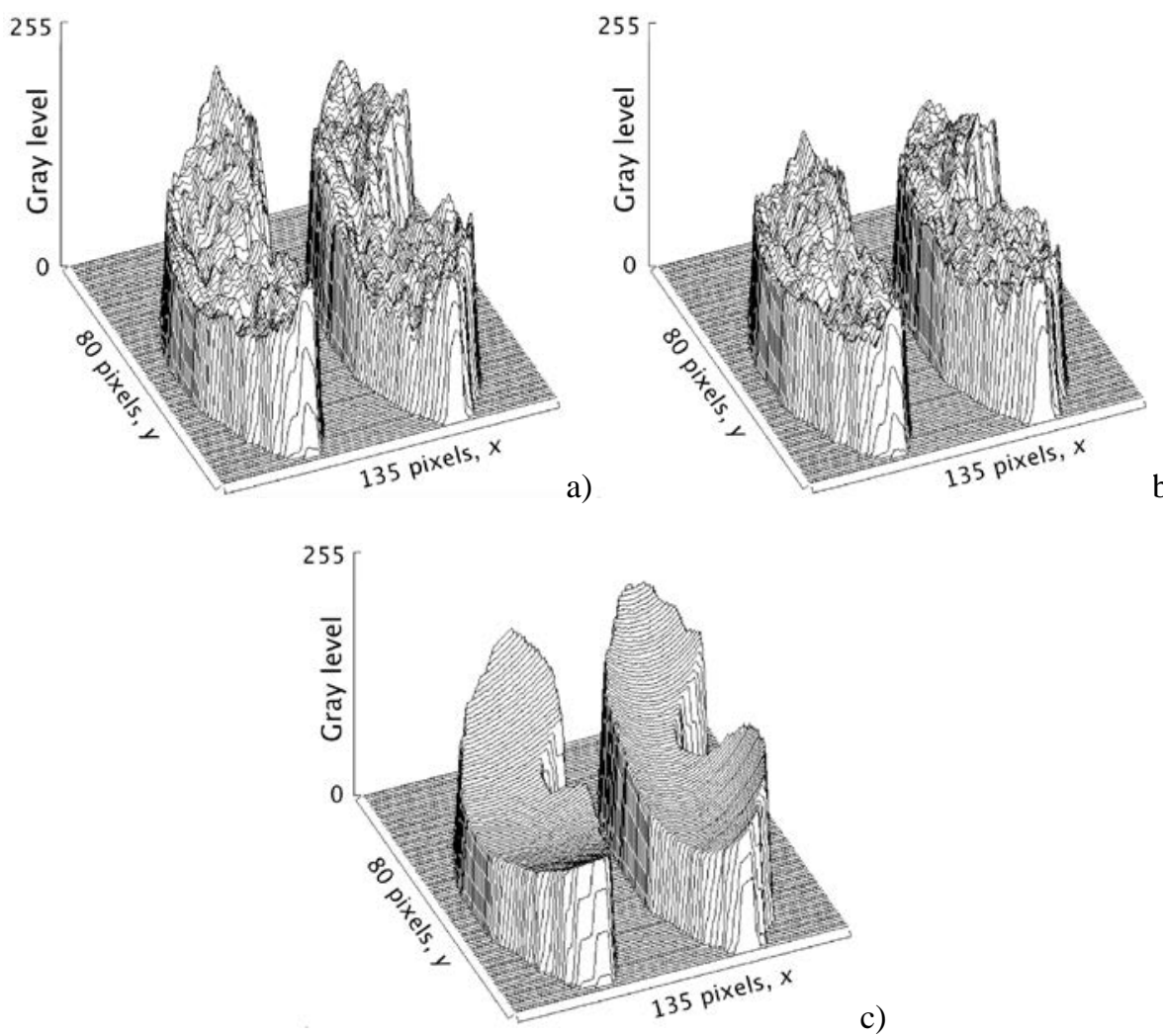

b)

c)

Figure 16. Surface plots of liver MR image gray levels (slice \#50) inside ROIs. Healthy organ (on the left) and cirrhotic tissue (on the right): a) original images (Fig. 1), b) corrected images (Fig. 9), c) additive correction field (Fig. 14)

\subsection{Classification of Brodatz textures}

Figure 17 shows distributions of GLCMs elements build for sample texture (shown in Fig. 3c) and its two distorted versions (Fig. 5) using image nonuniformity models described in Sections 2 and 3. Assumed distortions introduce linear changes of the modelled nonuniformity: $m_{k}$ ranging in $[-80,80]$ and $v_{k}$ in $[0.4,1.6]$ for the additive and multiplicative case, respectively. Although the nonuniformities introduced to the images with these example ranges are not perhaps small, the assumed variations more effectively visualize their effects on the parameters of real-world textures. Moreover, ROIs taken from the images shown in Fig. 5 for texture analysis were of lower size - 60x60 pixels. Total variations of local intensity and contrast within those ROIs were accordingly smaller than for the full-size images.

It can be observed that variable intensity extends the GLCM elements along its diagonal when compared to its original distribution (Fig. 17b). For contrast variations (Fig. 17c), larger scatter of elements outside matrix diagonal is observed as a result of multiplication of element indexes by function from Fig. 4b. However, if most of GLCM elements will be 
located close to its diagonal (which seems to happen for majority of natural textures) and smooth contrast variations are assumed, the scatter will be not significant. Thus resulting matrix will be similar to this obtained for additive artifacts. Thus the same texture parameters whose values do not depend on translation along matrix diagonal should be robust to texture contrast variation.
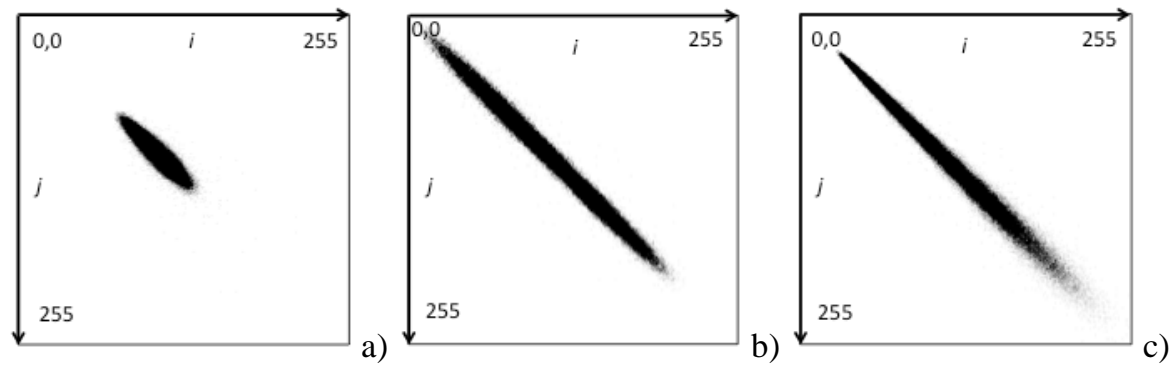

Figure. 17. GLCM (vertical direction, interpixel distance equal to 1) for texture from Fig. 3c (a), with additive (b) and multiplicative (c) nonuniformities

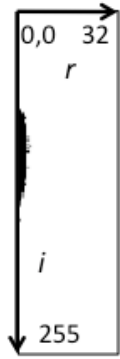

a)

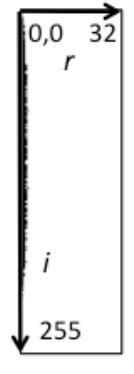

b)

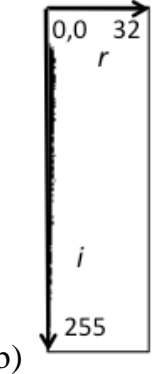

c)

Figure. 18. RL matrices (only first 32 columns shown, there are no longer runs) for texture from Fig. 3c (a) and for the additive (b) and multiplicative (c) artifact.

Sample RLMs for textures from Figs. 3c and 5 are presented in Fig. 18. Both types of degradation result in element scattering along intensity axis and their transition towards shorter runs. Following the discussion in Section 3, one can expect that all RLM derived parameters will be affected by both additive and multiplicative distortions. By definition, these parameters depend on a sum of all processed matrix elements. Thus increase of element number (resulted by their splitting) will alter evaluated RLM feature values. Especially features that additionally directly depend on the index that corresponds to run lengths (Short Run Emphasis, Long Run Emphasis, Fraction) will be strongly affected. In conclusion, no RLM based feature is insensitive to the discussed image nonuniformities.

Classification results of texture samples from Figs. 3 and 5 by means of a 1-NN classifier are shown in Fig. 19. The left column presents scatterplots of two most discriminant features selected according to Fisher criterion applied to original textures. They provide perfect classification, however for the distorted images misclassification takes place due to the spread of texture feature values caused by nonuniformity artifacts. The right column shows results obtained for two other parameters, less sensitive to image variable intensity and contrast. As can be observed, these features preserve their values both for additive and multiplicative degradation model, providing correct texture classification. 

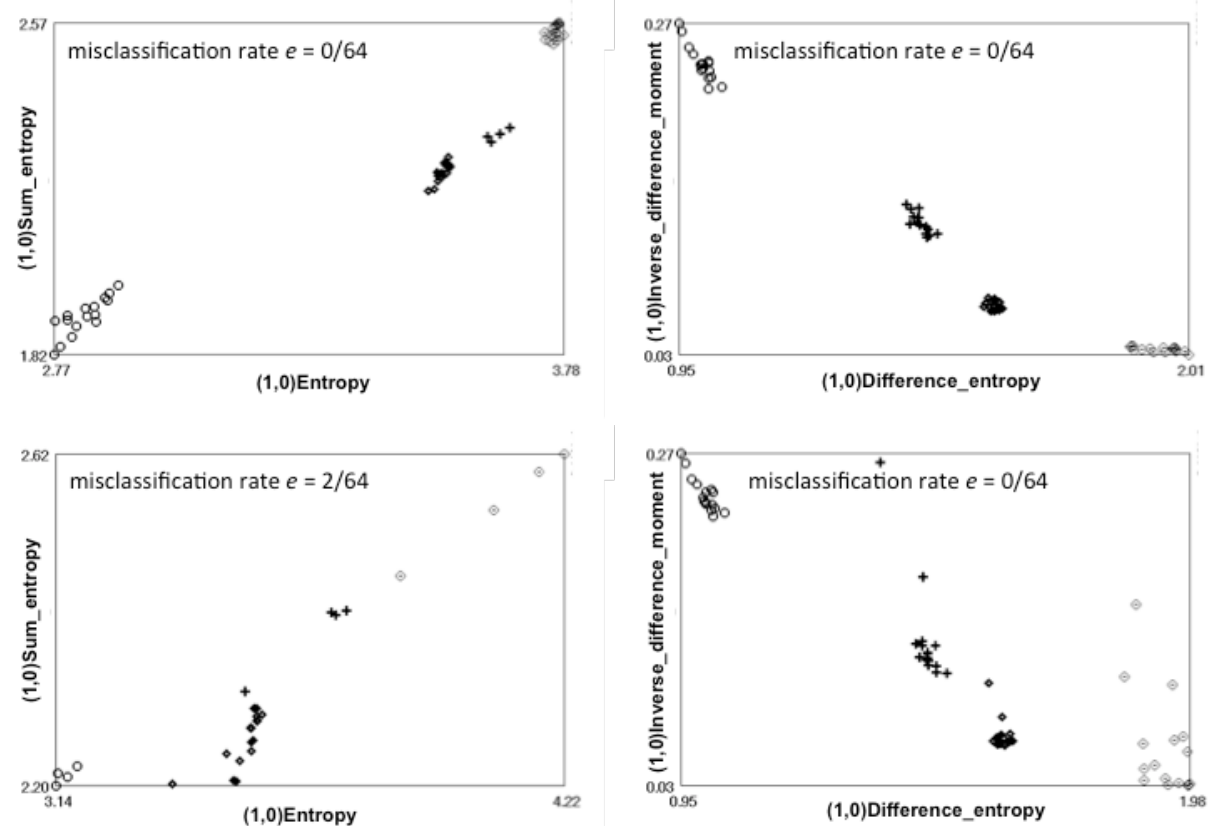

a)
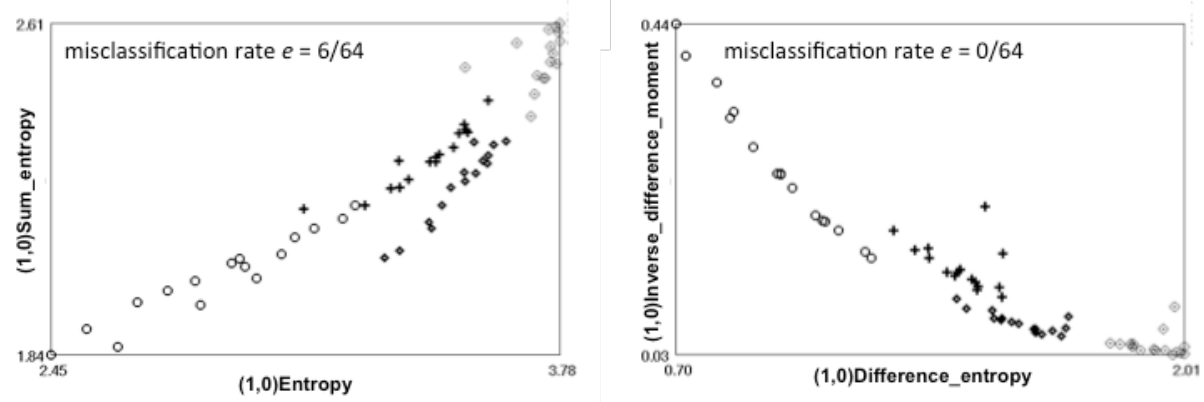

b)

Figure 19. Scatter plots of texture parameters used for classification of images from a) Fig. 3, b) Fig. 5a, and c) Fig. 5b. Distribution of features with highest Fisher coefficient is shown in the left column, while those less sensitive to image nonuniformities - in the right column. Symbols 'dotted circle', 'cross', 'circle', 'diamond' correspond to textures from Fig. 3a, b, c, and d, respectively.

Different results were obtained for classification of the same textures considering the RLM parameters only. Three parameters of largest F coefficient gave correct classification of the original textures. These parameters (as well as any other RLM-type subset) were unable to provide error-free discrimination of distorted images. Finally, Haar DWT based features were considered. Features calculated for subband obtained for at least one highpass filtration represented the highest $F$ coefficient, thus they were selected for classification. Introduced texture inhomogeneities did not modify their distribution, thus 
features selected for original textures provided also correct discrimination of the distorted ones. Results for classification of texture with multiplicative nonuniformities obtained for RLM and DWT features are shown in Fig. 20.

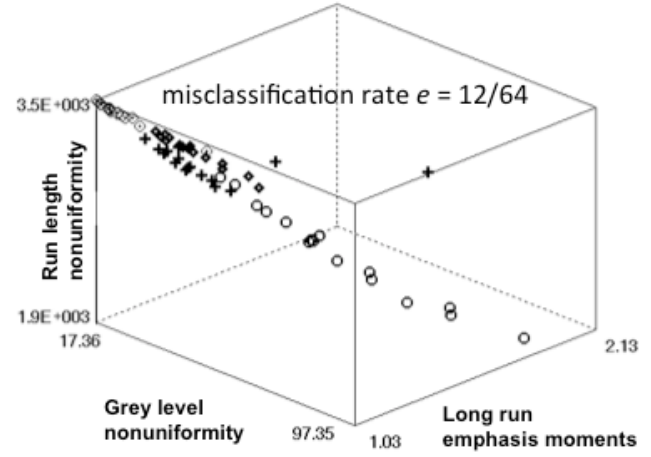

a)

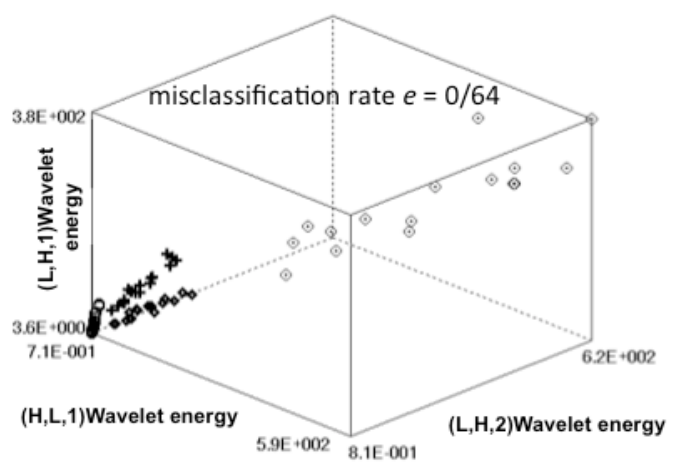

b)

Figure 20. Scatter plots of texture parameters used for classification of images from Fig. 5b using a) three RLM and b) three DWT features with the highest F coefficient. Symbols 'dotted circle', 'cross', 'circle', 'diamond' correspond to textures from Fig. 3a, b, c, and d, respectively.

\section{Discussion and Conclusions}

It has been demonstrated that results of quantitative texture analysis by means of the popular texture descriptors are sensitive to variations in local image intensity. These nonuniformity artifacts are especially prominent in real-life MRI where large image area/volume of uniform texture is considered. The presence of those artifacts may bring up misleading results to MRI texture analysis. This has been illustrated in Fig. 8 where apparent very good separation of texture classes was actually caused by image intensity inhomogeneity fields (Fig. 14), irrelevant to texture and structural tissue changes related to the disease development. This suggests, the smoothly varying intensity patterns, consistent within the image classes, were the primary properties that allowed the images discrimination. Referring back to the general characterization of image components [10], one can say that in the liver images under 
analysis, the smooth spatial tone variations are dominant over the texture. Moreover, each class of images has - for some technical reason - a typical tonal pattern.

Image local intensity and/or contrast variation may cause texture misclassification due to image brightness and/or contrast inhomogeneities. Texture parameters selected for nondistorted images might be unable to correctly discriminate the same images with these artifacts present.

In conclusion, correction of nonuniformity artifacts is recommended, followed by ROI normalization (e.g. with the “ \pm 3 sigma” scheme), prior to texture analysis. Two models, additive and multiplicative, were considered to show that such correction is feasible.

An attempt was made to identify texture features which, in some extent, are robust to image nonuniformities. It has been discussed and demonstrated that some features derived from GLC matrix possess this property, as parameters estimated with Haar DWT also do. It is expected that presented results will help in texture feature selection and new feature definition, especially in the cases where correction of image nonuniformity cannot be performed.

\section{References}

[1] Altunbas M. C. et al., A post-reconstruction method to correct cupping artifacts in cone beam breast computed tomography, Med Phys, 34, 7, 2007, 3109-3118.21

[2] Amadasun M., King R., Textural features corresponding to textural properties, IEEE Trans Syst Man Cybernetics, 19, 1989, 1264-1274.4

[3] Bahl G. et al., Noninvasive classification of hepatic fibrosis based on texture parameters from double contrast-enhanced magnetic resonance image, J Magn Res Imaging, 36, 2012, 1154-1161.10

[4] Belaroussi B., Milles J., Carme S., Zhu J-M., Benoit-Cattin H., Intensity nonuniformity correction in MRI: Existing methods and their validation, Med Image Anal, 10, 2006, 234-246.14

[5] Brodatz P., Textures - A Photographic Album for Artists and Designers, Dover, 1966.24

[6] Castelano G., Bonilha L., Li L-M., Cendes F., Texture analysis of medical images, Clin Radiology, 59, 2004, 1061-1069.1

[7] Gu J., Ramamoorthi R., Belhumeur P., Nayar S., Removing image artifacts due to dirty camera lenses and thin occluders, SIGGRAPH Asia, 2010.20

[8] Hajek M., Dezortova M., Materka A., Lerski R. (eds.), Texture analysis of magnetic resonance imaging, EU COST B21, Prague, Med4Publishing, 2006.8

[9] Haralick R. M., Shanmugam K., Dinstein I., Textural features for image classification, IEEE Trans Syst Man Cybern, 3, 1973, 610-621.7

[10] Haralick R. M., Statistical and structural approaches to texture, Proc IEEE, 67, 1979, 786-804.17

[11] http://docs.scipy.org/doc/scipy-0.14.0/reference/tutorial/optimize.html, accessed on 22 December, 2014.23

[12] Kassner A., Thornhill R., Texture analysis: a review of neurologic MR imaging applications, Am J Neuroradiol, 31, 2010, 809-816.2

[13] Lespessailles E., et al., Clinical interest of bone texture analysis in osteoporosis: a case control multicenter study, Osteoporosis Int, 19, 2008, 1019-1028.6 
[14] Levine M. D., Vision in man and machine, New York, Mc-Graw-Hill, 1985.3

[15] Li X-Z., Williams S., Bottema M.J., Background intensity independent texture features for assessing breast cancer risk in screening mammograms, Pattern Rec Letters, 34, 2013, 1053-1062.16

[16] Madabhushi A., Feldman M. D., Metaxas D. N., Tomaszewski J., Chute D., Automated detection of prostatic adenocarcinoma from high-resolution ex-vivo MRI, IEEE Trans Med Imaging, 24, 2005, 1611-1625.15

[17] Mallat S.G., Multifrequency channel decompositions of images and wavelet models, IEEE Trans. on Acoustics, Speech, and Signal Processing, 37, 1989, 2091-2110.26

[18] Materka A., Strzelecki M., Lerski R., Schad L., Feature evaluation of texture test objects for magnetic resonance imaging, in: M. K. Pietikainen (editor), Texture Analysis in Machine Vision, Series in Machine Perception \& Artificial Intelligence, Singapore, World Scientific, 40, 2000, 197-206.11

[19] Materka A., Strzelecki M., On the Importance of MRI Nonuniformity Correction for Texture Analysis, Proc. of IEEE SPA 2013, 26-28 September 2013, Poznan, Poland, 118-123.22

[20] Materka A., Strzelecki M., Texture analysis methods-a review, Brussels, EU COST B11 Report, 1998. Available at: http://eletel.eu/programy/cost/pdf_1.pdf. Last accessed on 22 December, 2014.18

[21] Rao A. R., Lohse G. L., Towards a texture naming system: Identyfying relevant dimensions of texture, Vision Research, 36, 11, 1996, 1649-1669.5

[22] Schürman J., Pattern classification, John Wiley \& Sons, 1996. 19

[23] Strzelecki M., Materka A., On sensitivity of texture parameters to smooth variations of local image intensity and contrast, Proc. of IEEE SPA 2014, 22-24 September 2014, Poznan, Poland, 48-53.25

[24] Strzelecki M., Szczypiński P., Materka A., Klepaczko A., A software tool for automatic classification and segmentation of 2D/3D medical images, Nucl Instrum Meth A, 702, 2013, 137-140.12

[25] Styner M., Van Leemput K., Retrospective evaluation and correction of intensity inhomogeneities, in: L. Landini, V. Positano, M. F. Santarelli (eds.), Advanced Image Processing in Magnetic Resonance Imaging, Boca Raton, Taylor \& Francis CRC Press, 2005, 145-168.13

[26] Szczypinski P., Strzelecki M., Materka A., Klepaczko A., MaZda - A software package for image texture analysis, Comp Meth Programs Biomed, 94, 2009, 66-76.9

This paper is a revised and extended version of work originally presented at the Signal Processing Algorithms, Architectures, Arrangements, and Applications Conference, 22-24 September 2014, Poznan, Poland 